\title{
LEAST SQUARES APPROACH TO THE ALIGNMENT OF THE GENERIC HIGH PRECISION TRACKING SYSTEM
}

\author{
PAWEL BRÜCKMAN DE RENSTROM \\ University of Oxford, DWB, Keble Road, Oxford OX1 3RH, UK \\ and \\ Institute of Nuclear Physics PAN, ul. Rdzikowskiego 152, Kraków 31-342, PL \\ E-mail: p.bruckman1@physics.ox.ac.uk \\ STEPHEN HAYWOOD \\ Rutherford Appleton Laboratory, Chilton Didcot OX11 OQX, UK \\ E-mail:S.Haywood@ral.ac.uk
}

\begin{abstract}
A least squares method to solve a generic alignment problem of a high granularity tracking system is presented. The algorithm is base on an analytical linear expansion and allows for multiple nested fits, e.g. imposing a common vertex for groups of particle tracks is of particular interest. We present a consistent and complete recipe to impose constraints on either implicit or explicit parameters. The method has been applied to the full simulation of a subset of the ATLAS silicon tracking system. The ultimate goal is to determine $\approx 35,000$ degrees of freedom (DoF's). We present a limited scale exercise exploring various aspects of the solution.
\end{abstract}

\section{Introduction}

The ultimate alignment precision of the modern HEP tracking systems can be achieved by means of a global $\chi^{2}$ fit of the alignment parameters to trajectories of real particles reconstructed in the detector. The advantage of the method is that it uses all the available information, and potentially can correct all possible misalignments without the need for iteration. However, in common with any algorithm based on reconstructed tracks, there are certain distortions of the detector which are difficult to correct. These arise from distortions which displace detector hits such that they still lie on helices. Among the most common ones are well known sagitta distortions (global: $R \delta \phi=\alpha+\beta R+\gamma R^{2}, \phi$ dependent: $\delta X=a+b R+c R^{2}$ and $\theta$ dependent: $\left.\delta \phi=\kappa R \cot (\theta)\right)$, so-called "telescope" $(\delta Z=e+f R)$ and various radial deformations (e.g. elliptical). These global distortions of the detector geometry, so called "weak modes", can lead to significant biases in the reconstructed track parameters. In this paper, we present the generic formalism to solve the least squares alignment problem as well as discuss various extensions leading to better control of the weak modes.

\section{The Formalism}

The alignment algorithm is based on the minimisation of the "global $\chi 2$ " defined as:

$$
\chi^{2}=\sum_{\text {tracks }} r^{T} V^{-1} r \quad \text { where } \quad r_{i} \equiv\left(\vec{m}_{i}-\vec{e}_{i}(\pi, a)\right) \cdot \hat{k}
$$

Here $\vec{e}_{i}$ denotes the $i$ 'th intersection point of the extrapolated track with a sensor plane and $\vec{m}_{i}$ is the position of the associated detector hit. $\hat{k}$ is the unit vector defining the measurement direction for the sensor plane ${ }^{a}$. The intersection point depends on the track parameters $(\pi)$ as well as on the subset of alignment parameters related to the intersected module $(a) . V$ is the covariance matrix of hit position measurements.

\subsection{The Basic Least Squares Linear Expansion}

The minimisation condition requires:

$$
\frac{d \chi^{2}}{d a}=0 \quad \Longrightarrow \quad \sum_{\text {tracks }} \frac{d r^{T}}{d a} V^{-1} r=0
$$

The assumption about the corrections being small allows us to use a linear expansion throughout. In particular, all second order derivatives are neglected. The expansion reads:

$$
\sum_{\text {tracks }} \frac{d r^{T}}{d a_{0}} V^{-1}\left(r_{0}+\frac{d r}{d a_{0}} \delta a\right)=0
$$

from which the generic solution can be obtained:

$$
\delta a=-\left(\sum_{\text {tracks }} \frac{d r^{T}}{d a_{0}} V^{-1} \frac{d r}{d a_{0}}\right)^{-1} \sum_{\text {tracks }} \frac{d r^{T}}{d a_{0}} V^{-1} r_{0}
$$

where $r_{0}$ is the initial residual and $\frac{d r}{d a_{0}} \stackrel{(4)}{\equiv}$ $\left.\frac{d r}{d a}\right|_{\pi=\pi_{0}, a=a_{0}}$. Because $r$ is a function of $\pi$ and $a$

\footnotetext{
${ }^{a}$ Recall that for a pixel detector, each physical hit corresponds to two distinct measurements (2D) and therefore gives rise to two residuals along the two measurement directions.
} 
the full derivative from Eq. 4 can be written as:

$$
\frac{d r}{d a}=\frac{\partial r}{\partial a}+\frac{\partial r}{\partial \pi} \frac{d \pi}{d a}
$$

where $\frac{d \pi}{d a}$ is obtained by differentiating the solution from a single track fit. In exact analogy to 4 , we get:

$$
\delta \pi=-\underbrace{\left(\frac{\partial r^{T}}{\partial \pi_{0}} V^{-1} \frac{\partial r}{\partial \pi_{0}}\right)^{-1}}_{J} \frac{\partial r^{T}}{\partial \pi_{0}} V^{-1} r\left(\pi_{0}, a\right)
$$

Combining 4, 5 and 6 yields the solutions for the alignment parameters alone:

$$
\delta a=-\underbrace{\left(\sum_{\text {tracks }} \frac{\partial r^{T}}{\partial a_{0}} W \frac{\partial r}{\partial a_{0}}\right)^{-1}}_{\mathcal{M}} \underbrace{\sum_{\text {tracks }} \frac{\partial r^{T}}{\partial a_{0}} W r\left(\pi_{0}, a_{0}\right)}_{\mathcal{V}}
$$

where

$$
W \equiv V^{-1}-V^{-1} \mathrm{E}^{-1} \mathrm{E}^{T} V^{-1},\left.\quad \mathrm{E} \equiv \frac{\partial r}{\partial \pi}\right|_{\pi=\pi_{0}}
$$

$\mathcal{M}$ is a symmetric $n \times n$ matrix and $\mathcal{V}$ a vector of size $n$, where $n$ is the number of alignment DoF's. Formula 7 can be shown to be equivalent to the one obtained using purely matrix manipulation methods ${ }^{1}$.

\subsection{Fitting a Common Event Vertex}

In order to include a vertex fit into the formalism, we have to redefine the track parameterisation. Only three perigee parameters survive $(\pi=$ $\left.\pi\left(\phi, \cot \theta, Q / p_{\mathrm{T}}\right)\right)$. Impact parameters are replaced by the common vertex for the event $\left(b=\left(x_{b}, y_{b}, z_{b}\right)\right)$. Definition of the residuals (Eq. 1) takes the new form:

$$
r_{i} \equiv\left(\vec{m}_{i}-\vec{e}_{i}(\pi, b, a)\right) \cdot \hat{k}
$$

The generic solution from Eq. 4 still holds, however, the full derivative takes a more complicated form:

$$
\begin{aligned}
& \frac{d r}{d a}=\frac{\partial r}{\partial a}+\mathrm{E} \frac{d \pi}{d a}+\mathrm{F} \frac{d b}{d a} \\
& \frac{d \pi}{d a}=-J^{-1} \mathrm{E}^{T} V^{-1}\left(\frac{\partial r}{\partial a}+\mathrm{F} \frac{d b}{d a}\right) \\
& \frac{d b}{d a}=-\underbrace{\left(\sum_{\text {tracks }}^{e v} \mathrm{~F}^{T} W \mathrm{~F}\right)^{-1}}_{M_{b}}\left(\sum_{\text {tracks }}^{e v} \mathrm{~F}^{T} W \frac{\partial r}{\partial a}\right)
\end{aligned}
$$

where we additionally defined $\mathrm{F} \equiv \frac{\partial r}{\partial b}$. Despite the above complexity, the final solution can be written in a compact form:

$$
\delta a=-\underbrace{\left(\sum_{\text {tracks }} \frac{\partial r^{T}}{\partial a_{0}} X \frac{\partial r}{\partial a_{0}}\right)^{-1}}_{\mathcal{M}} \underbrace{\sum_{\text {tracks }} \frac{\partial r^{T}}{\partial a_{0}} X r\left(\pi_{0}, b_{0}, a_{0}\right)}_{\mathcal{V}}
$$

where we used the relation:

$$
\frac{d r}{d a}=X \frac{\partial r}{\partial a}, \quad X \equiv W-W F M_{b}^{-1}\left(\sum_{\text {tracks }}^{e v} \mathrm{~F}^{T} W\right)
$$

\subsection{Adding External Constraints}

Consider an example of constraints on track parameters. In general, they may be non-linear, however they have to be linearised before they can enter the formalism. Constraints appear as extra bi-linear terms in the expression for $\chi^{2}$ :

$$
\chi^{2}=\sum_{\text {tracks }}\left(r^{T} V^{-1} r+(\pi-x)^{T} S^{-1}(\pi-x)\right)
$$

where vector $x$ and covariance matrix $S$ define the constraint on $\pi$. The solution for the track parameters is given by:

$$
\begin{gathered}
\delta \pi=-J^{-1}\left(\mathrm{E}^{T} V^{-1} r\left(\pi_{0}, a\right)+S^{-1}\left(\pi_{0}-x\right)\right) \\
J \equiv \mathrm{E}^{T} V^{-1} \mathrm{E}+S^{-1}
\end{gathered}
$$

The solution for the alignment parameters can be derived in the usual way and yields:

$$
\begin{aligned}
& \delta a=-\mathcal{M}^{-1} \\
& \sum_{\text {tracks }}\left(\frac{\partial r^{T}}{\partial a_{0}} W r_{0}-\frac{\partial r^{T}}{\partial a_{0}} V^{-1} \mathrm{E}^{-1} S^{-1}\left(\pi_{0}-x\right)\right)
\end{aligned}
$$

where $\mathcal{M}$ is as in Eq. 11, but with $J$ as in Eq. 14 .

\section{Example Tests}

The above formalism was implemented in prototype code in order to test the alignment of the ATLAS Silicon Tracking System ${ }^{2}$. The entire system consists of 1744 pixel modules (2D readout, $14 \times 115 \mu \mathrm{m}$ resolution) and 4088 double-layer strip detector modules with two sides rotated by $40 \mathrm{mrad}$ stereo angle $(16 \times 580 \mu \mathrm{m}$ resolution $)$. Given 6 DoF's of every module, there are 34,992 parameters to be determined. Solution for the entire system presents a substantial numerical challenge and as such is beyond the scope of this report. Here we present results from a test setup consisting of a region of $0<\eta<1$. The selected subset of the system contains 1030 silicon modules (both pixel and strip) and corresponds to over $1 / 6$ of the entire tracking system (6180 DoF's). Only a limited data sample of 450,000 muon tracks $\left(2<p_{T}<20 \mathrm{GeV} / \mathrm{c}\right)$ was used, so the results do not represent the ultimate precision.

\subsection{The Baseline Algorithm}

Diagonalisation of the matrix $\mathcal{M}$ obtained from Eq. 7 yields in the eigenvalue spectrum shown in Figure 1. 
First four values are zero (up to the numerical accuracy) and correspond to unresolved translations and rotation w.r.t. the $Z$ axis of the entire system. ${ }^{b}$ To obtain meaningful results reciprocals of these four eigenvalues are set to zero which is equivalent to freezing these modes. The "weak modes" corre-
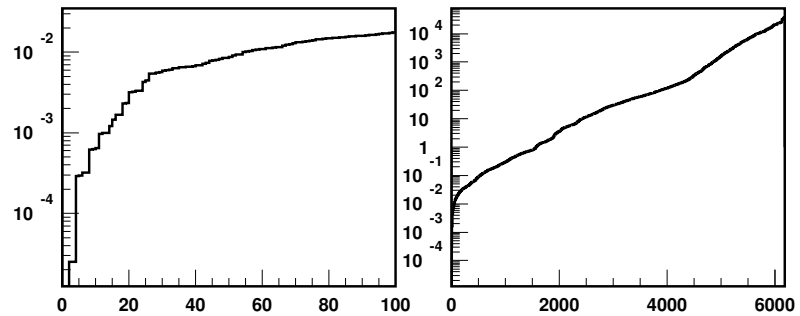

Figure 1. Eigen-spectrum of the matrix $\mathcal{M}$. Left plot zooms on the 100 weakest modes.

spond to the lowest (finite) eigenvalues and consequently dominate the overall error on the alignment parameters. ${ }^{c}$ More importantly, these global shape deformations lead directly to biases on fitted track parameters. Figure 2 shows pulls of the alignment corrections as determined from the perfectly aligned detector. The distribution is nicely Gaussian, centred at zero and the scatter plot does not reveal any suspicious structures. To further test the algorithm
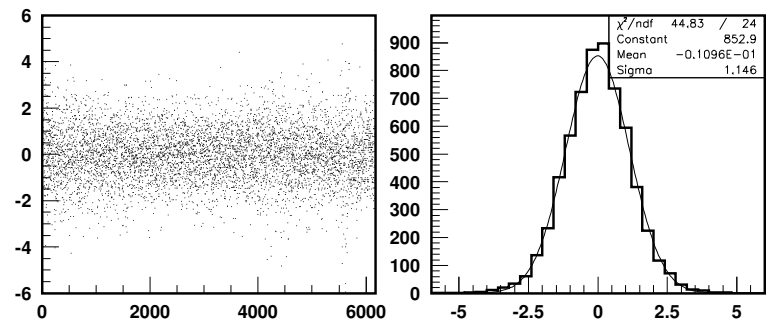

Figure 2. Pulls of the alignment parameters in the diagonal space as determined for the perfectly aligned detector.

we collectively shifted all pixel detectors by $\Delta X=$ $200 \mu \mathrm{m}, \Delta Y=100 \mu \mathrm{m}, \Delta Z=400 \mu \mathrm{m}$ in the ATLAS global frame ( $Z$ axis is parallel to the beam line). Tracks were refitted to the modified geometry and the alignment algorithm run. We observed no outstanding deformations to cylinders. In order to make the discussion more quantitative, we projected alignment parameters on rigid cylinders using the Jacobian transformation: $d r / d A_{l}=\left(d r / d a_{k}\right)\left(\partial a_{k} / \partial A_{l}\right)$, with $A_{l}$ being the $7 \times 6$ DoF's of the seven rigid cylin-

\footnotetext{
${ }^{b}$ The other two rotations do not result in singular modes due to the defined and fixed direction of the magnetic field.

${ }^{c}$ Recall that the error is proportional to square root of the reciprocal of the eigenvalue.
}

ders. Note that this simple technique may prove very useful as a day- 0 solution or a genuine method to reduce number of DoF's. Results are given in Table 1 . The solution settled on a minor "telescope" mode which is one of the weakest and most difficult to control. Otherwise, corrections in the orthogonal plane are consistent with the imposed misalignment within the statistical error.

Table 1. Corrections $(\mu \mathrm{m})$ to rigid cylinders.

\begin{tabular}{|lrrr|}
\hline cylinder & $\Delta X$ & $\Delta Y$ & $\Delta Z$ \\
PIX b-layer & $-198 \pm 5$ & $-105 \pm 5$ & $-450 \pm 29$ \\
PIX layer 1 & $-199 \pm 4$ & $-102 \pm 4$ & $-445 \pm 27$ \\
PIX layer 2 & $-200 \pm 3$ & $-101 \pm 3$ & $-440 \pm 25$ \\
SCT barrel 3 & $-2 \pm 3$ & $0 \pm 3$ & $-22 \pm 15$ \\
SCT barrel 4 & $-2 \pm 2$ & $0 \pm 2$ & $-16 \pm 10$ \\
SCT barrel 5 & $-1 \pm 1$ & $0 \pm 1$ & $-2 \pm 5$ \\
SCT barrel 6 & $0 \pm 0$ & $0 \pm 0$ & $0 \pm 0$ \\
\hline
\end{tabular}

\subsection{The Common Vertex Constraint}

Applying the common vertex constraint fit of Eq. 11 (there are $\approx 10$ muons per event in our data sample) yielded qualitatively similar results but the absolute error on the pixel module positions (close to the interaction point) was reduced by a factor of two. Figure $4 a$ shows the difference in the eigenvalues of the weak modes (first 100) after applying the vertex fit.

\subsection{Constraints on Track Parameters}

Starting from a perfect detector we imposed specific constraints on all track parameters: $\cot \theta^{\prime}=\cot \theta-$ $0.001, \sigma=0.0001$ and $Q / p_{T}^{\prime}=Q / p_{T}-0.01, \sigma=$ $0.001(\mathrm{GeV} / \mathrm{c})^{-1}$. These particular constraints were chosen as they directly correspond to well known weak modes, namely the "telescope" mode and the "sagitta" distortion. Alignment solution of Eq. 15 was determined with the above constraints imposed.
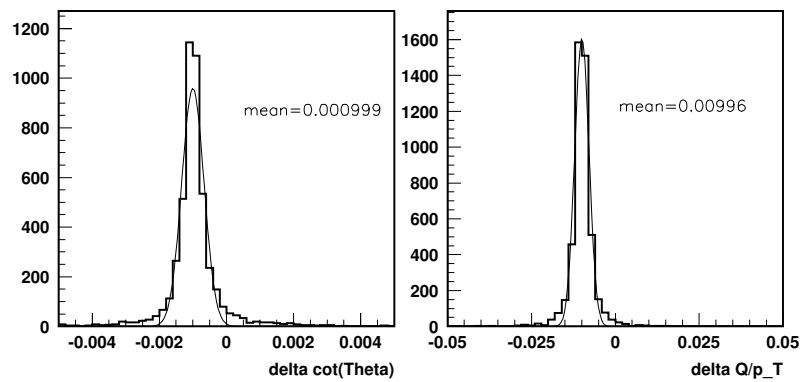

Figure 3. Change to track parameters after the refit to distorted detector geometry. See section 3.3 for more details.

Then, an independent track sample was refitted to the modified detector geometry. Figure 3 shows the 
resulting shifts to the track parameters. The deformation to the detector geometry led precisely to the required change of track parameters.

\subsection{Constraint on the Mass of a Resonance}

The idea of the constraints on track parameters can be extended to the constraint on the mass of a known resonance (e.g. $Z \rightarrow \mu^{+} \mu^{-}, J / \Psi \rightarrow \mu^{+} \mu^{-}$). All that is needed is an extra term in the $\chi^{2}$ expression:

$$
\chi^{\prime 2}=\chi^{2}+\left(m_{i}-M\right)^{T} \frac{1}{\sigma_{i}^{2}}\left(m_{i}-M\right)
$$

where $M$ is the known mass of the resonance and $\sigma_{i}$ its assumed width accounting for experimental resolution. The solution for the constrained fit is obtained using $d m / d a=(\partial m / \partial \pi)(d \pi / d a)$ :

$J^{\prime}=J+\frac{\partial m^{T}}{\partial \pi} \frac{1}{\sigma_{i}^{2}} \frac{\partial m}{\partial \pi}, \quad \mathcal{V}^{\prime}=\mathcal{V}+\frac{d m_{i}^{T}}{d a} \frac{1}{\sigma_{i}^{2}}\left(m_{i}^{0}-M\right)$

The idea was tested in a very naive way using the muon event sample. Tracks with $p_{T}>5 \mathrm{GeV} / \mathrm{c}$ were combined into pseudo-resonances if the resulting mass was $5 \mathrm{GeV} / \mathrm{c}^{2}$ or larger. The initial mass of the pair was used for the $M$ value in each case. $\sigma$ was set to $0.1 \mathrm{GeV} / \mathrm{c}^{2}$ for all pairs. The improvement of the sensitivity to weak modes is shown in Figure $4 b$. Results are encouraging but the method clearly deserves proper validation using true $Z$ and $J / \Psi$ samples.
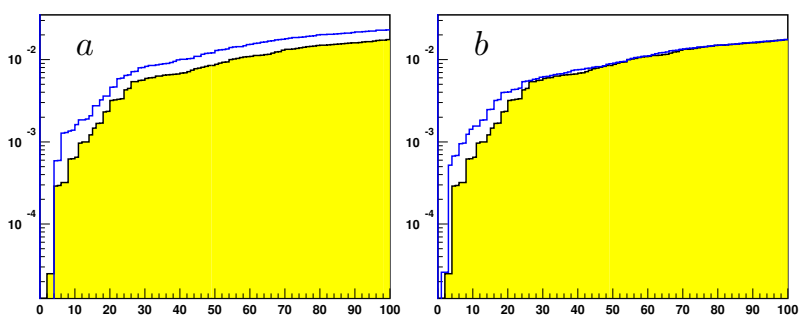

Figure 4. Eigen-spectrum for the baseline algorithm (shaded histogram) with (a) the vertex constraint and (b) mass constraint superimposed. Only 100 weakest modes shown.

\subsection{External Constraints on the Geometry}

External constraints may result from various mechanical considerations, actual hardware monitoring of the deformations of the support structure, etc. Whatever the source, they rarely determine positions of the individual modules. Instead, they give constraints in terms of arbitrary shape functions $F_{k}$. The extra term takes the form:

$$
\chi^{\prime 2}=\chi^{2}+p_{k} \frac{1}{\sigma_{k}^{2}} p_{k} \text { with } p_{k}=F_{k i} a^{i}-A_{k}
$$

where $A_{k}$ is the requested amplitude of the $k$ 'th shape function and $\sigma_{k}$ is the corresponding error.
The constraint results in the following extra contributions to the final big matrix $\mathcal{M}$ and vector $\mathcal{V}$ :

$$
\mathcal{M}^{\prime}=\mathcal{M}+\frac{1}{\sigma_{k}^{2}}(\underbrace{\hat{F}_{k} \hat{F}_{k}^{T}}_{\text {tensor }}), \quad \mathcal{V}^{\prime}=\mathcal{V}-\frac{1}{\sigma_{k}^{2}} A^{k} \hat{F}_{k}
$$

We tested the idea using directly two known weak modes, namely an "elliptical" and a "telescope" distortion. Table 2 shows the imposed constraint and the resulting amplitude of the corresponding mode after realignment. It was found that all other modes were unchanged relative to the unconstrained solution of section 3.1 .

Table 2. Imposed vs reconstructed constraints on the geometry (arbitrary units.)

\begin{tabular}{|lrr|}
\hline mode & "elliptical" & "telescope" \\
constrained $A$ & 1.0000 & 0.00000 \\
constrained $\sigma(A)$ & 0.0100 & 0.00100 \\
reconstructed $A$ & 0.9870 & 0.00007 \\
reconstructed $\sigma(A)$ & 0.0099 & 0.00100 \\
\hline
\end{tabular}

\section{Summary}

The least squares solution to the alignment of large HEP tracking systems has been presented. It has the potential to incorporate various extra constraints to improve its sensitivity to weak modes. Preliminary tests of the proposed extensions using the ATLAS silicon tracking system have been presented.

\section{Acknowledgements}

We would like to thank the ATLAS Inner Detector Alignment Group for helpful discussions. In particular, we acknowledge valuable discussions and crosschecks provided by Adlene Hicheur and thank him for his collaboration. The datasets used were generated using ATLAS software, for which we thank the ATLAS Software Team. This work had been inspired by the ideas used by ALEPH: ALEPH-97-116, A.Bonissent et al.

\section{References}

1. V. Blobel, C. Kleinwort, "A new method for the high-precision alignment of track detectors", Proceedings of the Conference on Advanced Statistical Techniques in Particle Physics, University of Durham, UK March 18-22, 2002.

2. ATLAS Collaboration, ATLAS Detector and Physics Performance TDR, ATLAS TDR 15, CERN/LHCC/99-15, 25 May 1999. 